\title{
Auf dem Weg zu mehr Transparenz bei den Einnahmen politischer Parteien aus Wirtschaftstätigkeit
}

- Eine Zwischenbilanz anhand der aktuellen Rechenschaftsberichte - ${ }^{1}$

\section{Einleitung}

Der Vermögensbestand und die Wirtschaftstätigkeit politischer Parteien sind in der öffentlichen und wissenschaftlichen Diskussion lange vernachlässigt worden. Das gleiche gilt für die Einnahmen der Parteien aus Vermögenserträgen und unternehmerischen Gewinnen, obgleich diese Einnahmen einen wesentlichen Bestandteil des Parteienfinanzierungssystems darstellen. Die insoweit geführte Debatte konzentrierte sich vielmehr lange Zeit ausschließlich auf die Parteispenden und die staatliche Parteienfinanzierung. Die Gründe für diese einseitige Sichtweise lagen wohl im vermeintlich geringen Anteil der Erträge aus Vermögen und Unternehmenstätigkeit an den Gesamteinnahmen der Parteien ${ }^{2}$ und darin, dass die Rechtslage den Parteien erlaubte, ihr Vermögen und ihre Wirtschaftstätigkeit im Dunklen zu halten. Im Zusammenhang mit der Parteispendenaffäre 1999-2000 wurde jedoch auch die Vermögensbildung und wirtschaftliche Betätigung politischer Parteien Gegenstand einer regen öffentlichen Auseinandersetzung. Nachdem die Praxis der Vermögensverschleierung der hessischen CDU in Einzelheiten bekannt geworden war und ein breites politisches und wissenschaftliches Echo ausgelöst hatte, ${ }^{3}$ sahen sich zahlreiche politische Akteure veranlasst, die Vermögensverhältnisse der Konkurrenzparteien in polemischer Weise zu thematisieren. Insbesondere die Unternehmensbeteiligungen der SPD wurden in Frage gestellt ${ }^{4}$. Die Bundestagsfraktionen der CDU/CSU und der FDP legten Gesetzentwürfe vor, die weitgehende Einschränkungen der wirtschaftlichen Betätigung der Parteien, insbesondere im Medienbereich, vorsahen. Diese scheiterten an der rot-grünen Mehrheit. ${ }^{5}$ Einige CDU-regierte Bundesländer initiierten restriktive Änderungen ihrer Landesmediengesetze bezüglich der Rundfunkbeteiligungen von Parteien. ${ }^{6}$

1 Der Erstverfasser ist freier Autor in Berlin. Der Zweitverfasser ist Staatsanwalt an der Staatsanwaltschaft Potsdam.

2 Vgl. BTDrs. 15/255, S. 4f.

3 Vgl. H. Merten, MIP 1999 Sonderbeilage, S. 11f.; J. Ipsen, JZ 2000, 685f.; BVerfG, DVBl. 2004, S. 1357f.

4 In den Medien wurde die SPD mit zahlreichen Vorwürfen bezüglich ihres Finanzsystems konfrontiert (vgl. Die Welt 13.03.2000 und 28.04.2000; Rheinischer Merkur 27.07.2001 und 14./15.03.2002). Auch in der Lehre fanden sich Stimmen, die weitgehende gesetzliche Einschränkungen der Vermögensbildung und -anlage von Parteien forderten, so P. M. Huber, Parteien in der Demokratie, in: Badura/Dreier (Hrsg.), Festschrift 50 Jahre Bundesverfassungsgericht, Bd. 2, Tübingen 2001, S. 609f.; A. Feser, Die politische Meinung 2003, S. 71f.; M. Möstl, DÖV 2003, S. 106f.

5 BTDrs. 14/7441; 15/3097.

6 Vgl. Art. 24 III Bayerisches Mediengesetz; § 13 Nr. 6 Landesmediengesetz Baden-Württemberg; § 6 II Nr. 4 Hessisches Privatrundfunkgesetz; § 6 III Niedersächsisches Mediengesetz. 
Schließlich kam es 2002 zu einer von allen Bundestagsfraktionen getragenen Novellierung des Parteiengesetzes. ${ }^{7}$ Eines ihrer Hauptziele war die Schaffung von mehr Transparenz, was den Bestand des Parteivermögens, die Anlage desselben und die Einnahmen der Parteien aus Vermögen und unternehmerischer Tätigkeit betrifft. ${ }^{8}$ Die im Juni 2005 erfolgte Veröffentlichung der Rechenschaftsberichte der Parteien für das Jahr $2003^{9}$ ist die erste nach dem novellierten Parteiengesetz und gibt Anlass zur Prüfung, inwieweit die neuen Normen sich in der Praxis bewährt haben. ${ }^{10}$

\section{Die Vorgaben des Grundgesetzes}

Aufgrund des Demokratiegrundsatzes wird eine umfassende Publizität ${ }^{11}$ der gesamten Tätigkeit der Parteien als Vermittler politischer Impulse zwischen Gesellschaft und Staat verlangt. ${ }^{12}$ Wegen des Zusammenhangs zwischen wirtschaftlicher und politischer Macht verpflichtet das Grundgesetz die Parteien, insbesondere über ihre Finanzverhältnisse öffentlich Rechenschaft zu geben (Art. 21 I 4 GG). Die Offenlegung der Parteifinanzen soll den Bürger in den Stand versetzen, Einflüsse, Abhängigkeiten und Interessen zu erkennen und seine Wahlentscheidung in Kenntnis dessen zu treffen. ${ }^{13}$ Dieser Zielsetzung diente auch die Novellierung des Art. 21 I 4 GG von 1983, ${ }^{14}$ mit der die Pflicht zur Rechnungslegung auf die Verwendung der Mittel und das Parteivermögen erweitert wurde. Das verfassungsrechtliche Transparenzgebot verpflichtet seither die Parteien, sichtbar zu machen, wer finanziell hinter ihnen steht, wie sie wirtschaften und schließlich welchen wirtschaftlichen Einfluss sie ihrerseits auf Dritte ausüben können. Nach Art. 21 I 4 GG unterliegen alle Finanzquellen der Parteien in gleicher Weise der Publizitätspflicht, so dass auch der Gesetzgeber zwischen ihnen

7 Achtes Gesetz zur Änderung des Parteiengesetzes vom 28. Juli 2002 (BGBl. I, S. 2268).

8 Begründung zum interfraktionellen Gesetzentwurf BTDrs. 14/8778, S. 14.

9 Die Veröffentlichung erfolgte bisher in zwei Bundestagsdrucksachen: 15/5550 für die im Bundestag vertretenen Parteien und 15/5551 für die übrigen Parteien mit Anspruch auf staatliche Parteienfinanzierung. Die Rechenschaftsberichte der sonstigen Parteien werden zu einem späteren Zeitpunkt bekannt gemacht.

10 Zur weitergehenden Frage der verfassungsrechtlichen Zulässigkeit von Vermögensbildung und unternehmerischer Tätigkeit politischer Parteien und von gesetzlichen Einschränkungen derselben vgl. umfassend M. Angelov, Vermögensbildung und unternehmerische Tätigkeit politischer Parteien, erscheint 2006.

11 Vgl. zum »Öffentlichkeitsprinzip« M. Morlok, in: Dreier, GG, Art. 21, Rn. 112; Art. 20, Rn. 72f.

$12 \mathrm{Zu}$ den Funktionen politischer Parteien D. Grimm, Politische Parteien, in: Benda/Maihofer/ Vogel, (Hrsg.), Handbuch des Verfassungsrechts der Bundesrepublik Deutschland, Berlin 1994, S. 605; M. Morlok, in: Dreier, GG, Art. 21, Rn. 21; K. Stern, Das Staatsrecht der Bundesrepublik Deutschland, München 1984, S. 457; F. Boyken, Die neue Parteienfinanzierung: Entscheidungsprozeßanalyse und Wirkungskontrolle, Baden-Baden 1998, S. 122.

13 Vgl. BVerfGE 24, 300 (356); 85, 264 (319). Ein berechtigtes Interesse an umfassenden Informationen über die wirtschaftlichen Verhältnisse einer Partei hat auch die politische Konkurrenz. Die übrigen Parteien können aus dem Wissen, wie sich eine Partei ökonomisch betätigt, Konsequenzen für ihre eigene politische Strategie ziehen und so zur öffentlichen Kontrolle beitragen.

14 BGBl. I (1983), S. 1481. 
nicht unterscheiden darf. ${ }^{15}$ Der Aufbau und die Anlage von Parteivermögen können ebenso wie die Spenden Dritter ein Risikopotential für die Beeinflussung politischer Entscheidungen bergen. Daher besteht von Verfassungs wegen die Pflicht zu einer gesetzlichen Regelung, die die Parteien wirksam zu einer weitgehenden Transparenz ihrer ökonomischen Tätigkeit anhält. Diese müssen jedenfalls ab einer bestimmten Höhe verpflichtet werden, ihre einzelnen Vermögensquellen gesondert auszuweisen und den Bestand und die Verwendung des Parteivermögens offen zu legen.

\section{Mängel der Rechtslage und Probleme der Praxis bis 2002}

\section{Geschichtlicher Abriss der Rechtslage}

Seinen verfassungsrechtlichen Regelungsauftrag ließ der Gesetzgeber lange unerfüllt. Vor Inkrafttreten des Parteiengesetzes wurden Angaben zum Parteivermögen und zu den Erträgen daraus nur vereinzelt und in sehr unzureichender Weise aus innerparteilichen Dokumenten und Presseberichten bekannt. Erstmals wurde die Rechnungslegungspflicht der Parteien im 5. Abschnitt des 1967 verabschiedeten Parteiengesetzes geregelt. Der Rechenschaftsbericht bestand lediglich aus einer Einnahmenrechnung, in der sämtliche Einnahmen, einschließlich solcher aus Vermögen, auszuweisen waren. ${ }^{16}$ Nach § 27 II PartG 1967 war bei den Einnahmen aus Vermögen und eigener wirtschaftlicher Betätigung lediglich der Reinertrag einzusetzen.

Als Reaktion auf den »Flick-Skandal ${ }^{17}$ und im Zusammenhang mit der Änderung des Art. 21 I 4 GG $^{18}$ wurde das Parteiengesetz 1983 neu gefasst. ${ }^{19}$ Damit sollte »eine neue Phase der finanziellen Rechnungslegung ${ }^{20}{ }^{20}$ politischer Parteien in Deutschland beginnen, da im Rechenschaftsbericht neben der Einnahmenrechnung nunmehr eine

15 Vgl. Rechtliche Ordnung des Parteiwesens, Bericht der vom Bundesminister des Innern eingesetzten Parteienrechtskommmission, Frankfurt am Main 1958, S. 205. A.A. die Mehrheit des Parteispenden-Untersuchungsausschusses und ein Teil der Lehre, die die Ungleichbehandlung von Parteivermögen und Parteispenden für sachlich gerechtfertigt halten, da letztere eine besondere Relevanz für die demokratische Willensbildung aufwiesen und ihnen für die Verwirklichung des Transparenzgebots ein größeres Gewicht zukomme als der Offenlegung von Einnahmen aus Vermögen und aus eigener wirtschaftlicher Tätigkeit. Vgl. BTDrs. 14/9300, S. 410; P. M. Huber, DÖV 2000, S. 745 (751).

16 Laut § 24 II PartG 1967 mussten folgende Posten gesondert ausgewiesen werden: 1. Mitgliedsbeiträge; 2. Beiträge der Fraktionsmitglieder und ähnliche regelmäßige Beiträge; 3. Einnahmen aus a) Vermögen, b) Veranstaltungen, Vertrieb von Druckschriften und Veröffentlichungen und sonstiger mit Einnahmen verbundener Tätigkeit der Partei; 4. Spenden; 5. Kredite; 6. Erstattung von Wahlkampfkosten; 7. Sonstige Einnahmen. Es wurde für die Parteien die Möglichkeit vorgesehen, dem Rechenschaftsbericht, insbesondere auch einzelnen seiner Posten, kurzgefasste Erläuterungen beizufügen (§ 24 III PartG 1967).

17 Vgl. dazu BTDrs. 10/5079.

18 Gesetz zur Änderung des Grundgesetzes, BGBl. I (1983), S. 1481.

19 Gesetz zur Änderung des Parteiengesetzes und anderer Gesetze vom 22. Dezember 1983 BGBl. I, S. 1577. Vgl. Bekanntmachung der Neufassung des Parteiengesetzes, BGBl. I (1984), S. 242f.

20 T. Kaufner, Rechenschaftspflicht und Chancengleichheit, in: G. Wewer (Hrsg.), Parteinfinanzierung und politischer Wettbewerb, Opladen 1990, S. 101. 
Ausgabenrechnung und eine Vermögensrechnung stehen mussten. In der Einnahmenrechnung mussten weiterhin die Einnahmen aus Vermögen und aus eigener Wirtschaftstätigkeit in zwei gesonderten Posten ausgewiesen werden (§ 24 II Nr. 2, 3 PartG 1984). Die Vermögensrechnung wurde in $§ 24$ IV PartG 1984 geregelt und enthielt eine wenig detaillierte Aufgliederung des Anlage- und Umlaufvermögens. ${ }^{21}$ Die nächste umfassende Novellierung des Parteiengesetzes 1993 brachte trotz entsprechender Vorschläge keine Neuerungen für den hier interessierenden Themenkreis. ${ }^{22}$

\section{Mängel der gesetzlichen Regelung über die Vermögenstransparenz.}

Die oben skizzierten Regelungen konnten einen umfassenden Einblick in die Vermögensverhältnisse der Parteien nicht sichern. Die Aussagekraft der 1983 eingeführten Vermögensrechnung war unzureichend, weil sie nicht genügend differenziert war, die Bilanzstrukturen der einzelnen Parteien sehr unterschiedlich waren und es an einheitlichen Bewertungsvorschriften fehlte. ${ }^{23}$ Nach wie vor fast völlig in Dunkel gehüllt blieben daher insbesondere das Immobilienvermögen und die Unternehmensbeteiligungen der Parteien.

Hinzu kam - hinsichtlich des Immobilienvermögens - ein weiteres Problem. Im Regelfall wurde die Verwaltung der Parteiimmobilien parteieigenen Vermögensverwaltungsgesellschaften und Vereinen übertragen, und zwar oft auch dann, wenn die jeweilige Parteigliederung als eingetragener Verein konstituiert und somit grundbuchfähig war und es daher rechtlich ohne weiteres möglich gewesen wäre, die Partei selbst als Eigentümer ins Grundbuch eintragen zu lassen. ${ }^{24}$ Das jeweilige Grundstück bzw. Gebäude erschien im Rechenschaftsbericht häufig nicht unter der Position »Haus- und Grundvermögen«, da die Immobilien als Betriebsvermögen der Vermögensverwaltungsgesellschaften angesehen wurden. In den Rechenschaftsberichten erschien lediglich - unter der Position »Finanzanlagen«, nicht unterschieden von anderen, »echten « Finanzanlagen - das Stammkapital dieser Vermögensverwaltungsgesellschaften, bzw., bei einer unter 100-\%-igen Parteibeteiligung, der Kapitalanteil der

21 Die Vermögensrechung umfasste: A. Besitzposten: I. Anlagevermögen: 1. Haus- und Grundvermögen; 2. Geschäftsstellenausstattung; 3. Finanzanlagen; II. Umlaufvermögen: 1. Beitragsforderungen; 2. Forderungen auf Erstattung von Wahlkampfkosten; 3. Forderungen auf Chancenausgleich; 4. Geldbestände; 5. sonstige Vermögensgegenstände; B. Schuldposten: I. Rückstellungen; II. Verbindlichkeiten: 1. Beitragsverbindlichkeiten; 2. Verbindlichkeiten gegenüber Kreditinstituten; 3. Sonstige Verbindlichkeiten; III. Reinvermögen (positiv oder negativ). Die Saldierungsmöglichkeit des § 27 II PartG 1967 blieb in Bezug auf die Einnahmen aus Vermögen und aus Wirtschaftstätigkeit bestehen.

22 Der Änderungsantrag der Fraktion der Bündnis 90/Die Grünen, der die Erweiterung der Einnahmerechnung um drei Positionen vorsah, nämlich Mandats- und Amtsträgereinnahmen, Einnahmen aus Krediten und Einnahmen aus Unternehmenstätigkeit, wurde nicht berücksichtigt (vgl. BTDrs. 12/6139).

23 Vgl. dazu BTDrs. 14/4747, S. 53; 12/5575, S. 57; 13/140, S. 37; 13/8888, S. 58; 14/7979, S. 40.

24 Solche Gesellschaften und Vereine sind z.B. das SPD-Unternehmen »Konzentration $\mathrm{GmbH} \ll$, der »Hausverein der CDU e.V.«, der »Vermögensverwalterverein der Bundespartei Bündnis 90/Die Grünen e.V.«u.a. 
Partei an der Gesellschaft. ${ }^{25}$ Dadurch entstand ein Transparenzverlust, da die Vermögensverwaltungsgesellschaft einer Partei oft vermögender war als es ihrem nominellen Kapital entsprach und dies dem Rechenschaftsbericht nicht zu entnehmen war. Außerdem wurden die Vermögensverwaltungsorganisationen in den Rechenschaftsberichten namentlich nur selten genannt. ${ }^{26}$

Die Lage hinsichtlich der Transparenz der Unternehmensbeteiligungen der Parteien war kaum besser. Diese durften nämlich zusammen mit anderen Vermögensgegenständen (Wertpapiere, langfristige Forderungen usw.) dem Rechnungsposten »Finanzanlagen « (§ 24 IV Nr. 1 I 3 PartG a.F.) zugeschlagen werden. Dementsprechend blieben sie im Grunde unerwähnt. ${ }^{27}$ Verborgen blieben so außerdem die Struktur der Parteibeteiligungen und die benutzten rechtlichen Konstruktionen. Insbesondere war den Rechenschaftsberichten nicht zu entnehmen, ob und in welchem Ausmaß die Parteien sich Treuhändern bedienten. ${ }^{28}$

Die Aussagekraft der Rechnungsposten in der Einnahmenrechnung, die die Einnahmen aus Vermögen und wirtschaftlicher Tätigkeit widerspiegeln sollten, war ebenfalls gering. Die Aufgliederung der Einnahmenrechnung bezüglich der aus der Vermögensverwendung resultierenden Einnahmen in zwei Positionen ( $\$ 24$ II Nr. 4 und 5 PartG a.F.) erwies sich als unzureichend. Insbesondere bei Einnahmen aus unternehmerischer Tätigkeit ließ sich nicht eindeutig bestimmen, ob diese unter »Einnahmen aus Vermögen« oder »sonstiger mit Einnahmen verbundener Tätigkeit« auszuweisen waren. ${ }^{29}$ Defizite bestanden auch bei der Offenlegung der Mieteinnahmen, die bald als »Einnahmen aus Vermögen«, bald als »sonstige Einnahmen« ausgewiesen wurden. Zur Verschleierung der realen Vermögenslage der Parteien führte auch die in § 27 II 1

25 Vgl. BTDrs. 10/4104 (neu), S. 106; 11/5993, S. 102; 11/8130, S. 100.

26 Während die Grünen im Erläuterungsteil ihrer Rechenschaftsberichte regelmäßig weitgehende Angaben zu ihren Vermögensverwaltungsvereinen machten (vgl. BTDrs. 14/2508, S. $108 ; 14 / 5050$, S. $109 ; 15 / 2800$, S. 95), legten die übrigen Parteien nur gelegentlich Einzelheiten ihres Haus- und Grundvermögens offen (vgl. für die CDU BTDrs. 15/700, S. 57, für die PDS BTDrs. 15/700, S. 135 und für die FDP BTDrs. 15/2800, S. 134.). Die SPD als größte Immobilieneigentümerin unter den Parteien wies darauf hin, dass ihr Grundvermögen »aus grundbuchrechtlichen Gründen im Treuhandvermögen des SPD-Parteivorstandes, das von der Firma Konzentration GmbH verwaltet wird, erfasst « wird (vgl. BTDrs. 10/4104 neu, S. 106; 11/3315, S. 96; 11/5993, S. 102; 11/8130, S. 100), darüber hinaus finden sich nur einige sporadische und unvollständige Angaben, etwa zur »Bürohaus Berlin Stresemannstr./Wilhelmstr. mbH«, der die Parteizentrale in Berlin gehört (vgl. BTDrs. 15/700, S. 25; 15/2800, S. 26, vgl. auch Focus 32/2001, S. 20f.).

27 Parteiunternehmen wurden nur sporadisch bei konkreten Anlässen und zur Erläuterung anderer Positionen in den Rechenschaftsberichten erwähnt. Vgl. BTDrs. 11/3315, S. 96; 11/ 5993, S. 102; 14/5050, S. 83.

28 Die meisten Parteien setzen, allerdings teilweise bedingt durch ihre zivilrechtliche Stellung als nichtrechtsfähige Vereine, Treuhänder ein. Vgl. § 47 II Statut der CDU; § 25 I 1 Organisationsstatut der SPD; OLG Frankfurt am Main, NJW 1952, S. 792; K. Endlich, Recht und Praxis der politischen Parteien in der Bundesrepublik Deutschland, München 1992, S. 126.

29 Besondere Brisanz erhielt diese Problematik, als die Ausweisung der Gewinnausschüttungen der SPD-Holdinggesellschaft DDVG an die Partei im Rechenschaftsbericht für 1998 Gegenstand politischer und wissenschaftlicher Auseinandersetzung wurde. Vgl. dazu BTDrs. 14/4747, S. 31; 14/7979, S. 22; 14/9300, S. 470. 
i.V.m. § 26 II PartG a.F. geregelte Saldierungsmöglichkeit, wobei nicht klar geregelt war, welche Einnahmen mit welchen Ausgaben saldiert werden durften, was zu einer uneinheitlichen Rechnungslegungspraxis führte. ${ }^{30}$

Die Rechtslage bezüglich der Offenlegung der Vermögensverhältnisse der Parteien entsprach also den verfassungsrechtlichen Erfordernissen nicht. Der Gesetzgeber sah sich nach dem Aufflammen der öffentlichen Auseinandersetzung zum Handeln veranlasst und verabschiedete Regelungen, die die bestehenden Transparenzlücken beseitigen sollten.

\section{Rechtslage und Praxis seit 2002}

\section{Rechtslage seit 2002}

Mit der Novellierung des Parteiengesetzes $2002^{31}$ wurde die Rechenschaftspflicht der Parteien über ihr Vermögen und ihre Wirtschaftstätigkeit und die Erträge daraus erweitert. Der Notwendigkeit einer höheren Transparenz des Parteivermögens wollte der Gesetzgeber durch eine umfassende Änderung des § 24 PartG $^{32}$ Rechnung tragen, der den Rechenschaftsbericht regelt. ${ }^{33}$ Die Anforderungen an die Einnahmenrechnung ${ }^{34}$ wurden erhöht, indem nunmehr die Einnahmen aus Unternehmenstätigkeit und Beteiligungen, ${ }^{35}$ aus sonstigem Vermögen sowie aus Veranstaltungen und sonstiger mit Einnahmen verbundener Tätigkeit jeweils gesondert auszuweisen sind (§ 24 IV Nr. 5, 6 und 7). Ebenso wurde das Schema für die Ausgabenrechnung um eine Position zur Ausweisung von Ausgaben für Vermögensverwaltung erweitert (§ $24 \mathrm{~V} \mathrm{Nr.} \mathrm{2d).} \mathrm{Die}$ umstrittene Saldierungsmöglichkeit (§ 26 II 2 und § 27 II 1 PartG a.F.) wurde ersatzlos

30 Von dieser Saldierungsmöglichkeit machten alle Parteien regelmäßig Gebrauch. Vgl. BTDrs. 14/5050, S. 70, 82, 108, 133, 156; 14/8022, S. 39. Zur umstrittenen Handhabung der genannten Regelung seitens der SPD, die in ihrem Rechenschaftsbericht für das Jahr 1998 die Aufwendungen für den Bau der neuen Parteienzentrale in Berlin mit den Ausschüttungen der SPD-Unternehmen DDVG verrechnete, vgl. R. Wend/A. Albrecht-Baba, ZRP 2001, S. 561 (564); B. Küstermann, Die Rechenschaftspflicht der politischen Parteien, Osnabrück 2002, S. 163; BTDrs. 14/4747, S. 31; 14/7979, S. 22. Zu bemerken ist auch, dass das Parteiengesetz keine Sanktion bei fehlerhafter Ausweisung von Vermögen vorsah. Unrichtigkeiten in der Vermögensrechnung konnten lediglich zum Verlust des Anspruchs auf staatliche Parteienfinanzierung führen, §§ 19 IV, 23 IV PartG a.F.

31 Gesetz zur Änderung des Parteiengesetzes vom 28. Juli 2002, BGBl. I, S. 2268.

32 Soweit nicht ausdrücklich anders bezeichnet, verstehen sich §§-Angaben in diesem Beitrag als solche des Parteiengesetzes in seiner Fassung von 2004.

33 Vgl. Begründung zum gemeinsamen Gesetzentwurf der Fraktionen der SPD, CDU/CSU, Bündnis 90/Die Grünen und FDP, BTDrs. 14/8778, S. 19; Beschlussempfehlung und Bericht des Innenausschusses, BTDrs. 14/8824, S. 2.

34 Der Rechenschaftsbericht muss wie bisher aus einer Einnahmen-, Ausgaben- und Vermögensrechnung bestehen. Letztere wird als Vermögensbilanz bezeichnet und durch einen Erläuterungsteil ergänzt ( $\$ 24$ I 1 PartG).

35 Beteiligungen im Sinne des Parteiengesetzes sind Anteile gemäß § 271 I des HGB (§ 24 VII Nr. 2 S. 3 PartG), also Anteile an anderen Unternehmen, die bestimmt sind, dem eigenen Geschäftsbetrieb durch Herstellung einer dauernden Verbindung zu jenen Unternehmen zu dienen. 
gestrichen, so dass nunmehr alle Einnahmen, also auch solche aus Vermögen und aus Unternehmensbeteiligungen, mit ihrem vollen Betrag anzugeben sind (§ 26 II). ${ }^{36}$ Detaillierte Anforderungen an den jetzt obligatorischen Erläuterungsteil der Vermögensbilanz stellt nunmehr $§ 24$ VII. Nach dieser Vorschrift muss in den Erläuterungen eine Auflistung der Parteibeteiligungen an Unternehmen sowie der unmittelbaren und mittelbaren Beteiligungen dieser Unternehmen ihrerseits, jeweils mit Name und Sitz sowie unter Angabe des Anteils und der Höhe des Nominalkapitals, enthalten sein $(§ 24$ VII Nr. 1). Bei Parteibeteiligungen an Medienunternehmen sind auch deren Haupterzeugnisse zu benennen (Nr. 3).

Mit den neu eingeführten Regelungen über die Bewertung von Vermögensgegenständen wird ein weiterer Mangel des Parteiengesetzes beseitigt. Unternehmensbeteiligungen sind nun ausdrücklich zum Nominalwert auszuweisen ( $\$ 24$ VII Nr. 1). Weiterhin verlangt $\S 24$ VII Nr. 3 von den Parteien, im Abstand von fünf Jahren eine Bewertung des Haus- und Grundvermögens sowie der Unternehmensbeteiligungen nach dem Bewertungsgesetz vorzunehmen. ${ }^{37}$

Trotz dieser umfangreichen Novellierung sah der Gesetzgeber weiterhin Änderungsbedarf, ${ }^{38}$ insbesondere im Bereich der Rechnungslegung, ${ }^{39}$ und verabschiedete Ende 2004 die bisher letzte Novelle des Parteiengesetzes. ${ }^{40}$ Dabei handelt es sich aber eher um Detailkorrekturen. ${ }^{41}$

36 Bemerkenswert ist auch die Neuregelung des § 27 II bezüglich der sonstigen Einnahmen der Parteien. Diese sind künftig aufzugliedern und zu erläutern, wenn sie bei einer der in § 24 III aufgeführten Gliederungen mehr als 2\% der Summe der Einnahmen nach § 24 Abs. 4 Nr. 1 bis 6 ausmachen. Diese Verschärfung der Offenlegungspflicht trifft allgemein auf Zustimmung, denn die entsprechende Position im Rechenschaftsbericht war jahrelang »Versteck « für zweifelhafte Vermögenseinnahmen, die verbucht als »sonstige Einnahmen « in das legale Rechnungssystem der jeweiligen Partei eingeführt wurden. Vgl. H. H. v. Arnim, DVBl. 2002, S. 1073 (1076); T. Streit, MIP 2003, S. 68 (70); C. Lenz, NVwZ 2002, S. 769 (772).

37 Relevant für die Transparenz des Parteivermögens, der unternehmerischen Tätigkeit von Parteien sowie der Erträge daraus sind auch die neuen materiellen und formellen Anforderungen an die Rechenschaftsberichte ( $\S 23 f$.) und die neuen Sanktionsnormen ( $\S 31$ bf.).

38 Nicht zuletzt wegen der im Abschlussbericht der vom ehemaligen Bundespräsidenten Rau eingesetzten Parteienfinanzierungskommission geäußerten Kritik bezüglich der Regelung des Buchführungssystems der Parteien (vgl. BTDrs. 15/3140, S. 16f.).

39 Vgl. Begründung des Gesetzentwurfs der Fraktionen SPD, Bündnis 90/Die Grünen und FDP (BTDrs. 15/4246, S. 1); Abschlussbericht der Rau-Parteienfinanzierungskommission (BTDrs. 15/3140, S. 15f.).

40 Neuntes Gesetz zur Änderung des Parteiengesetzes vom 22. Dezember 2004 (BGBl. I, S. 3673).

41 Durch die Neufassung des § 24 I 1 PartG wird von den Parteien nunmehr ausdrücklich eine Zwei-Komponenten-Verbundrechnung verlangt, womit der Rechenschaftsbericht der Parteien dem handelsrechtlichen Jahresabschluss angenähert wird. Die Neufassung des § 24 II 1 PartG präzisiert den bisherigen Verweis auf die Bilanzvorschriften des HGB, die Gliederungsstruktur des Rechenschaftsberichts wird ebenfalls verbessert. 
2. Erste wirkliche Einblicke in die Wirtschaftstätigkeit der Parteien - Die Rechenschaftsberichte für das Jahr 2003

a) $S P D$

Bekanntlich ist die SPD die vermögendste und unternehmerisch aktivste deutsche Partei. ${ }^{42}$ In ihren Rechenschaftsberichten weist die SPD regelmäßig ein höheres Reinvermögen als alle übrigen Parteien zusammen aus. ${ }^{43}$ Aufschlussreiche Angaben über die Struktur ihres Vermögens und ihrer Unternehmensbeteiligungen waren aber jahrelang nur schwer zugänglich. Im Rechenschaftsbericht für 2003 setzt die SPD ihre seit dem Aufflammen des Parteispendenskandals 1999/2000 bestehende Praxis, Angaben über ihre Unternehmensbeteiligungen zu veröffentlichen, fort. ${ }^{44}$ Nach zahlreichen Umstrukturierungen besteht der SPD-Unternehmensbereich seit Mitte der 90-er Jahre aus zwei Ebenen. An der Konzernspitze steht die »Deutsche Druck- und Verlagsgesellschaft $\mathrm{mbH} \ll(\mathrm{DDVG}) .{ }^{45}$ 99,25\% der DDVG-Anteile werden von der SPD-Bundesschatzmeisterin als Treuhänderin der Partei, die restlichen $0,75 \%$ von der »Solidarität Verwaltungsund Treuhandgesellschaft $\mathrm{mbH}$ « gehalten. ${ }^{46}$ Die DDVG ist direkt und mittelbar an zahlreichen Unternehmen beteiligt, die in unterschiedlichen Branchen, vorwiegend im Medienbereich, tätig sind und die zweite Stufe des SPD-Unternehmensnetzes bilden. ${ }^{47} \mathrm{Be}-$ deutsame SPD-Unternehmen außerhalb der DDVG-Holding sind beispielsweise die »Konzentration $\mathrm{GmbH} \ll^{48}$ die »Verwaltungsgesellschaft Bürohaus Berlin Stresemannstraße/Wilhelmstraße mbH« sowie einige lokale Grundstücksverwaltungsgesellschaften.

42 Zur geschichtlichen Entwicklung der unternehmerischen Aktivitäten der SPD vgl. U. Danker u.a., Am Anfang standen Arbeitergroschen. 140 Jahre Medienunternehmen der SPD, Bonn 2003; G. Wewer, Sozialdemokratische Wirtschaftsbetriebe, Opladen 1987.

43 Vgl. BTDrs. 15/255, S. 20.

44 Auskunft über die Struktur des SPD-Unternehmensnetzes sowie über die Höhe der Unternehmenserträge kann in den letzten Jahren den veröffentlichten Geschäftsberichten der DDVG, den Berichten der Parteischatzmeisterin sowie Presseberichten entnommen werden.

45 Das Stammkapital der Gesellschaft beträgt 6.135.502,57 €, AG Berlin-Charlottenburg, HRB 83297.

46 Die »Solidarität Verwaltungs- und Treuhandgesellschaft $\mathrm{mbH}$ « hat ein Stammkapital von $51.129 €$. Alleingesellschafterin ist auch hier die SPD-Bundesschatzmeisterin treuhänderisch für die Partei, AG Bonn, HRB 2821, Bl. 147. Die Gesellschaft ist unternehmerisch nicht aktiv, sondern hält lediglich aus steuerrechtlichen Gründen neben der Schatzmeisterin Anteile an verschiedenen SPD-Unternehmen.

47 Diese Unternehmen sind im SPD Rechenschaftsbericht 2003 ausführlich aufgelistet, BTDrs. 15/5550, S. 60f. Die bedeutsamsten Unternehmen im Medienbereich sind »Dresdner Druckund Verlagshaus GmbH\& Co. KG«, »Druck- und Verlagshaus Frankfurt am Main GmbH« (Frankfurter Rundschau), »Druck- und Verlagsanstalt Neue Presse GmbH« (Coburg), »Dr. Erich Madsack GmbH« (Hannover), »Frankenpost Verlag GmbH« (Hof), »Oliva Druckund Verlagsgesellschaft $\mathrm{mbH}$ «(Cuxhaven), »Tivola Verlag GmbH« (Berlin), im Handelsbereich »Image Ident Marketing $\mathrm{GmbH}$ « (Berlin) und »Hamburger Pressepapier-Vertriebsgesellschaft $\mathrm{mbH}$ « (Hamburg), im Bereich Tourismus »SPD-Reiseservice $\mathrm{GmbH}$ « und »Reisebüro im Willy-Brandt-Haus GmbH« (beide Berlin), im EDV-Bereich »Office Consult Betriebswirtschaftliche Büroorganisation Beratungsgesellschaft $\mathrm{mbH}$ « (Berlin).

48 Diese traditionsreiche Gesellschaft ist heute vorrangig mit der Verwaltung von Immobilienvermögen befasst, worauf noch näher eingegangen wird. 
Die Struktur des SPD-Unternehmensnetzes sowie die für das Halten der Beteiligungen benutzte Rechtskonstruktion sind aber aus dem Parteirechenschaftsbericht alleine nicht ersichtlich, sondern können nur durch Heranziehen von Handelsregisterakten und innerparteilichen Dokumenten entschlüsselt werden. Beim Ausweis der mittelbaren Unternehmensbeteiligungen z.B. ist augenfällig, dass nicht klar gemacht wird, welche der mittelbaren Beteiligungen welchen Unternehmen zuzuordnen sind, an denen die SPD bzw. die DDVG beteiligt ist. ${ }^{49}$ Diese Zuordnung wird zwar vom Parteiengesetz nicht ausdrücklich verlangt ( $\$ 24$ VII Nr. 1), würde jedoch dem Sinn des Gesetzes, nämlich der Herstellung weitgehender Transparenz, entsprechen. Dass die SPD die einzige Partei ist, die über geringfügige mittelbare Beteiligungen an Rundfunkunternehmen verfügt, ist dem Rechenschaftsbericht für 2003 ebenfalls nicht zu entnehmen. ${ }^{50}$ Auch dies steht im Einklang mit dem Gesetzeswortlaut, da $§ 27$ VII Nr. 1 lediglich die Angabe der im Jahresabschluss der Parteiunternehmen aufgeführten Beteiligungen verlangt - und die geringfügigen Beteiligungen sind nach § 271 I 3 HGB darin nicht auszuweisen.

In Erfüllung der mit $§ 24$ VII Nr. 2 auferlegten Pflicht listet die SPD in ihrem Rechenschaftsbericht für 2003 erstmals die Hauptprodukte ihrer wichtigsten Medienunternehmen auf. ${ }^{51}$ Allerdings scheint diese Liste nicht vollständig zu sein. ${ }^{52}$

Hilfreich für die Einschätzung der Unternehmensbeteiligungen der SPD sind die im Erläuterungsteil des Rechenschaftsberichts ausführlich dargestellten Bewertungsmethoden. Bemerkenswert ist aber, dass die Bewertung und der Ausweis der Unternehmensbeteiligungen pauschal erfolgen, so dass der Wert einzelner Unternehmen und somit die Schwerpunkte des Beteiligungsportfolios nicht ersichtlich werden. ${ }^{53}$ Ähnlich werden - auch insoweit durchaus im Einklang mit dem Gesetzeswortlaut die Einnahmen aus Unternehmensbeteiligungen nur global ausgewiesen. ${ }^{54}$

49 Die Zuordnung der mittelbaren Beteiligungen der DDVG bzw. ihrer Tochterunternehmen ist aber ihrem Konzernabschluss sowie ihrem Konzernlagebericht für das Geschäftsjahr $2003 \mathrm{zu}$ entnehmen. Vgl. AG Berlin-Charlottenburg, HRB 83297 sowie die ausführliche Darstellung bei A. Feser, Der Genossen-Konzern. Parteivermögen und Pressebeteiligungen der SPD, München 2002, S. $76 f$.

50 Sie wurden in den Geschäftsberichten der DDVG aufgelistet. Vgl. DDVG, Geschäftsbericht 2003, S. 30. Zu den Rundfunkbeteiligungen der SPD vgl. auch A. Feser, Politische Studien 2002, S. 74f.

51 Vgl. BTDrs. 15/5550, S. 62.

52 So sind beispielsweise die Zeitungen »Nordbayerischer Kurier« und »Westfälische Rundschau « nicht benannt, obwohl die DDVG an ihren Verlagsgesellschaften indirekte Beteiligungen hält (vgl. F. Böckelmann, Wem gehören die Zeitungen, Konstanz 2000, S. 83 und 290; DDVG, Geschäftsbericht 2002, S. 10). Auch die Medienprodukte der »Tivola Verlag $\mathrm{GmbH}$ « (überwiegend Bücher für Kleinkinder, Computerspiele und Lernsoftware), an der die DDVG im Frühjahr 2003 75,25\% der Anteile erworben hatte, blieben im Erläuterungsteil des SPD-Rechenschaftsberichts unerwähnt (vgl. Die Welt 20. u. 21.03.2003; Pressemitteilung der DDVG vom 10.03.2003).

53 Der Wert der Unternehmensbeteiligungen der SPD zum Stichtag 01.01.2003 wird mit 165.257.923,- $€$ angegeben (vgl. BTDrs. 15/5550, S. 63).

54 Einnahmen aus Unternehmenstätigkeit und Beteiligungen sind beim Bundesparteivorstand (6.134.903 €), beim Landesverband Berlin (65.894 €) und beim Landesverband Bayern (4.200€) ausgewiesen. Vgl. BTDrs. 15/5550, S. 40f. 
Schon aus früheren Rechenschaftsberichten war bekannt, dass die SPD eine große Immobilieneigentümerin ist. ${ }^{55}$ Die meisten der SPD gehörenden Grundstücke und Gebäude werden von der »Konzentration $\mathrm{GmbH}$ « treuhänderisch verwaltet. Diese Gesellschaft ist auch selbst Eigentümerin von einigen Immobilien. ${ }^{56}$ Andererseits sind nicht alle SPD-Immobilien in der »Konzentration $\mathrm{GmbH}$ « zusammengefasst. Neben ihr bestehen lokale Grundstücksgesellschaften, die als Treuhänderinnen von Parteiuntergliederungen fungieren. An den meisten dieser Grundstücksgesellschaften ist allerdings wiederum die »Konzentration $\mathrm{GmbH} «$, gelegentlich zusammen mit der »Solidarität Verwaltungs- und Treuhandgesellschaft $\mathrm{mbH}$ « oder mit SPD-Funktionären, beteiligt. Welche diese Gesellschaften sind, konnte den Rechenschaftsberichten lange nicht entnommen werden. Im Rechenschaftsbericht 2003 sind sie erstmals namentlich aufgelistet. ${ }^{57}$ Die Bewertung des Haus- und Grundvermögens erfolgt gemäß $§ 24$ VII Nr. 3 pauschal, d.h. der Wert einzelner Immobilien erhellt nicht. Mangels ausdrücklicher gesetzlicher Verpflichtung fehlen im Rechenschaftsbericht auch Angaben dazu, welche konkreten Immobilien von den einzelnen Grundstücksgesellschaften verwaltet werden und welches die Höhe der Mieteinnahmen ist, die der SPD zu Gute kommen. ${ }^{58}$

Im Erläuterungsteil des Rechenschaftsberichts für 2003 finden sich fragmentarische Angaben über bisher nicht aufgeführte Kassen und Vermögenswerte der »Jungsozialisten in der SPD «, wobei der Eindruck erweckt wird, dass die Geldbestände der »Jusos« als Teilvermögen der Partei behandelt werden. ${ }^{59}$ Insgesamt wird aber nicht klar, welche finanziellen Beziehungen zwischen der Jugendorganisation und der Mutterpartei bestehen bzw. ob das Vermögen der »Jusos« insgesamt vom Parteienrechenschaftsbericht umfasst wird oder nicht. ${ }^{60}$

55 Die Zahl der treuhänderisch für den Parteivorstand gehaltenen Immobilien betrug z.B. 2000 71 und 200288 (vgl. BTDrs. 14/8022, S. 38; 15/2800, S. 26). Im Rechenschaftsbericht für 2003 ist die Zahl der SPD gehörenden Immobilien nicht angegeben.

56 Finanzbericht der SPD-Bundesschatzmeisterin 1997/99, S. 12. Nach Medienberichten soll die »Konzentration $\mathrm{GmbH}$ « bei mindestens sieben Grundstücken in Bonn, Hof, Heilbronn, Krefeld, Coburg, Hameln und Troisdorf nicht Treuhänderin, sondern selbst Eigentümerin sein und sie an SPD-Untergliedrungen zu unter dem Marktpreis üblichen Mietzinsen vermietet haben. Vgl. Focus 32/2001, S. 24; Die Welt 06.08.2001.

57 Z.B. »Grundstücksverwaltungsgesellschaft Wedding $\mathrm{mbH}$ «(Berlin), »Haus- und Grundstücksverwaltungsgesellschaft Hellweg $\mathrm{GmbH}$ « (Dortmund), »Offenbacher Abendblatt Grundstücksbesitzgesellschaft mbH« u.a. (vgl. BTDrs. 15/5550, S. 59). Zur Gesellschafterstruktur und den Geschäftsergebnissen dieser Unternehmen vgl. A. Feser (Fußn. 48), S. 61f.

58 Der Gesamtwert des Haus- und Grundvermögens der SPD zum Stichtag 31.12.2003 wird mit 92.006.500,- $€$ angegeben, vgl. BTDrs. 15/5550, S. 63.

59 Vgl. BTDrs. 15/5550, S. 70f.

60 Die ergänzende Erläuterung im Erläuterungsteil des SPD-Rechenschaftsberichts (BTDrs. $15 / 5550$, S. 56) bezieht sich lediglich auf die den »Jusos« zugewandten staatlichen Mittel (vgl. § 24 XII PartG). 


\section{b) $C D U$}

Auch die CDU verfügt über beachtliches Vermögen und über eigene Unternehmen, die primär in der Medienbranche und in der Immobilienverwaltung tätig sind. ${ }^{61} \mathrm{Im}$ Rechenschaftsbericht für 2003 sind die CDU-Unternehmen erstmals namentlich angegeben. $^{62}$ Das zentrale CDU-Unternehmen ist die »Union Betriebs $\mathrm{GmbH} «$ (UBG) mit Sitz in Rheinbach. Die Stammeinlage der UBG in Höhe von 2.150.000,- $€$ wird gemeinsam vom Bundesgeschäftsführer und vom Finanzbeauftragten der CDU treuhänderisch für die Partei gehalten. ${ }^{63}$ Die UBG gibt die meisten Publikationen der CDUBundespartei heraus und ist auch im Bereich der neuen Kommunikationstechnologien tätig. Ihre Hauptprodukte sind im Erläuterungsteil des Rechenschaftsberichts für 2003 ausführlich dokumentiert. ${ }^{64}$ Das Unternehmensnetz der CDU wird durch weitere Gesellschaften ergänzt, die der Partei wirtschaftlich meistens zu $100 \%$ gehören. ${ }^{65}$ Die konkreten Beteiligungsverhältnisse dieser Unternehmen sind dem Rechenschaftsbericht aber nicht zu entnehmen. ${ }^{66}$

Der Gesamtwert der Unternehmensbeteiligungen ist in der Bilanzrechnung des CDU-Rechenschaftsberichts für 2003 mit 1.497.610,62 € beziffert, wobei für die Bundespartei 1.201.638,17 € ausgewiesen sind. Einzeln bewertet sind lediglich die Beteiligungen an der »Pars-Verlag $\mathrm{GmbH} «(98.680,-€)$ und an der »Verlag für Landespolitik und Werbung $\mathrm{GmbH} \ll(113.915,-€){ }^{67}$

Im Unterschied zur SPD veröffentlicht die CDU auch die Bewertung einzelner Immobilien. ${ }^{68}$ Dies sind aber keinesfalls alle der Partei wirtschaftlich gehörenden Immobilien. Relevant in diesem Zusammenhang ist ein Satz im ersten Teil der Erläuterungen zum Rechenschaftsbericht, wonach »das Vermögen, sowie die Einnahmen und Ausgaben von Vereinen, welche treuhänderisch für Parteigliederungen Grundstücke und Gebäude halten, in dem Rechenschaftsbericht der jeweiligen Parteigliederung er-

61 Das Reinvermögen der CDU betrug zum 31.12.2001 57.961.382,- $€$, zum 31.12.2002 57.314.747,- $€$ und zum 31.12.2003 74.370.675,28 € (vgl. BTDrs. 15/2800, S. 34; 15/5550, S. 4).

62 Vgl. BTDrs. 15/5550, S. 26f.

63 AG Bonn, HRB 10605, Bl. 100.

64 Diese sind »Wirtschaftsbild «, »UiD - Union in Deutschland «, »Frau\&Politik«, »Rathaus consult« und »Forum Neue Länder«, vgl. BTDrs. 15/5550, S. 27.

65 Vgl. BTDrs. 15/5550, S. 26.

66 So enthalten beispielsweise die Erläuterungen zur Vermögensbilanz eine Bemerkung, dass $40 \%$ der Anteile an der »Kommunal-Verlag GmbH« mittelbar von der »Union Betriebs$\mathrm{GmbH}$ « gehalten werden, BTDrs. 15/5550, S. 27. Dies bedeutet für den unvoreingenommenen Leser, dass die restlichen Anteile sowie die Anteile an den übrigen aufgelisteten Unternehmen von der Parteiorganisation selbst gehalten werden, was aber nicht der Fall ist. Die weiteren Anteile an der »Kommunal-Verlag $\mathrm{GmbH}$ « werden in Wahrheit von der »Kommunalpolitischen Vereinigung der CDU und CSU Deutschlands e.V.« (36\%), der »Kommunalpolitischen Vereinigung der CDU Nordrhein-Westfalen e.V.« (18\%), der »Kommunalpolitischen Vereinigung der CSU Bayern e.V.« (3\%) und der CSU-Stadtratsfraktion München (3\%) gehalten, AG Recklinghausen, HRB 4175, Bl. 87.

67 BTDrs. 15/5550, S. 29.

68 BTDrs. 15/5550, S. 28. Der Gesamtwert des Haus- und Grundvermögens der CDU beträgt 2003 48.938.056, 51 €, BTDrs. 15/5550, S. 4. 
fasst $\ll$ sind ${ }^{69}$ Diese Immobilienverwaltungsvereine werden namentlich nicht angegeben, womit die Struktur eines großen Teils des Parteivermögens im Dunklen bleibt. Ein solcher Verein ist z.B. der »Hausverein der CDU e.V.«, der Eigentümer der CDUParteizentrale in Berlin ist. Wie bei der SPD enthält der CDU-Rechenschaftsbericht keine aufschlussreichen Angaben über eventuell zufließende Mieteinnahmen.

Eine Besonderheit der CDU, die von Bedeutung auch für ihre Finanzen ist, ist die Tatsache, dass die Partei über ein breites Netz von Umfeldorganisationen verfügt. Diese Vereinigungen sind gemäß $\S 38$ und 39 des CDU-Statuts, wo sie auch namentlich aufgezählt werden, konstitutive Teile der Gesamtpartei. Dies sollte bedeuten, dass ihr Vermögen sowie ihre Einnahmen und Ausgaben der Partei zuzurechnen sind und im Rechenschaftsbericht erfasst werden müssen. Die Praxis der CDU dürfte auch in diesem Sinne sein, ohne dass sie sich ausdrücklich hierzu verhält. Einige CDU-Vereinigungen haben eigene Unternehmen bzw. sind Mitgesellschafter in Gesellschaften, an denen CDU-Parteigliederungen Anteile halten, was aber dem Rechenschaftsbericht nicht zu entnehmen ist. So ist beispielsweise die »Kommunalpolitische Vereinigung der CDU des Landes Nordrhein-Westfalen e.V. « Alleingesellschafterin der »Kommunalpolitischen Vereinigung Dienstleistungs- und Beratungsgesellschaft $\mathrm{mbH}$ « (Recklinghausen $)^{70}$ und die »Christlich-Demokratische Arbeitnehmerschaft« hält die Anteile an der $\mathrm{CDA}$ Verlagsgesellschaft $\mathrm{mbH} \ll\left(\right.$ Siegburg) ${ }^{71}$

c) $C S U$

Im CSU-Rechenschaftsbericht für 2003 werden zwei Parteiunternehmen - „Bavaria Werbe- und Wirtschaftsdienste $\mathrm{GmbH}$ « (München) und »Union Report $\mathrm{GmbH}$ « (Nürnberg) ausgewiesen. ${ }^{72}$ Ihre Bewertung wird getrennt angegeben. Eine Besonderheit der Wirtschaftstätigkeit der CSU stellt die Herausgabe der Zeitschrift »Bayernkurier« dar. Sie wird im Rahmen eines parteieigenen Betriebes herausgegeben, d.h. der Verlag hat keine eigene Rechtspersönlichkeit. ${ }^{73}$ Die Einnahmen des Verlags werden daher als »Einnahmen aus Veranstaltungen, Vertrieb von Druckschriften und Veröffentlichungen « (§ 24 IV Nr. 7) ausgewiesen.

Bemerkenswert ist, dass die Ausweisung und die Bewertung des Haus- und Grundvermögens ausführlich und differenziert erfolgen und die Bewertungsmethoden dargestellt werden. ${ }^{74} \mathrm{Ob}$ die CSU auf Treuhänder oder Vermögensverwaltungsvereine zurückgreift, kann dem Rechenschaftsbericht nicht entnommen werden. ${ }^{75}$ Bezüglich der Ausweisung der Mieteinnahmen bestehen ähnliche Defizite wie bei der SPD und CDU. Hinsichtlich der Umfeldorganisationen ist zu bemerken, dass die meisten Verei-

69 BTDrs. 15/5550, S. 25.

70 AG Recklinghausen, HRB 5089, Bl. 14.

71 AG Siegburg, HRB 6670.

72 BTDrs. 15/5550, S. 148.

73 Im CSU-Rechenschaftsbericht für 2003 wird darauf ausdrücklich hingewiesen (BTDrs. 15/ 5550, S. 148).

74 BTDrs. 15/5550, S. 149, 151.

$75 \mathrm{Zu}$ den von der CSU Anfang der 90-er Jahre benutzten Treuhandkonstruktionen Endlich (Fußn. 28), S. 141. 
nigungen »gemeinsame« Teilorganisationen der CDU und CSU sind, weshalb insoweit auf IV.2.b. zu verweisen ist.

\section{d) FDP}

Die FDP weist regelmäßig das geringste Vermögen unter den im Bundestag vertretenen Parteien aus und ist die einzige Partei, die in der Vermögensbilanz ihres Rechenschaftsberichts für 2003 ein negatives Reinvermögen aufweist. ${ }^{76}$ Dies hängt mit der fast permanenten Überschuldung der Bundespartei und einiger Landesverbände zusammen. $^{77}$

Die Parteiunternehmen der FDP sind erstmals im Rechenschaftsbericht für 2003 bekannt gemacht worden. ${ }^{78}$ Die meisten von ihnen agieren in der Medienbranche und gehören zur Universum-Verlagsgruppe, deren zentrales Unternehmen »Universum $\mathrm{GmbH}$ « (Berlin) zu 100\% der FDP gehört. ${ }^{79}$ Weitere bedeutsame Medienunternehmen, an denen die FDP über die »Universum $\mathrm{GmbH}$ « beteiligt ist, sind u.a. »Liberal Verlag GmbH« (Berlin), »Universum Verlagsanstalt GmbH« (Wiesbaden), »Universum Verlagsanstalt GmbH \& Co. KG« (Wiesbaden). Auch eine beachtliche Anzahl von Medienunternehmen, an denen FDP-Landesverbände beteiligt sind (Niedersachsen Verlag GmbH, Hannover; Wirtschafts- und Sozialpolitik Verlag GmbH, Wiesbaden; L-W-D Liberale-Wirtschafts-Dienstleistungs GmbH, Düsseldorf) sowie von Unternehmen aus anderen Wirtschaftsbereichen, an denen die Bundespartei beteiligt ist (Liberal Vermögensverwaltungs-GmbH, Bonn; Liberal VermögensverwaltungsGmbH \& Co. Vermögensfonds KG, Bonn; ProLogo Gesellschaft für Veranstaltungsorganisation $\mathrm{GmbH}$, Bonn), sind zu erwähnen.

Ebenso wenig wie bei den anderen Parteien ist beim Ausweis der FDP-Beteiligungen ersichtlich, welchem Parteiunternehmen die mittelbaren Beteiligungen zuzuordnen sind und ob Treuhänder fungieren. ${ }^{80}$ In der Vermögensbilanz werden Unternehmensbeteiligungen beim Bundesverband sowie bei den Landesverbänden in Hessen, Niedersachsen und Nordrhein-Westfalen mit einem Gesamtwert von 3.879.201,75€ ausgewiesen. Einnahmen aus Unternehmenstätigkeit und Beteiligungen gibt die FDP 2003 nur beim Bundesverband (19.339,49 €) und beim Landesverband Nordrhein-Westfalen $(25.564,59 €)$ an. ${ }^{81}$ Hilfreich für die Einschätzung der Bedeutung der FDP-Unternehmen ist die im Erläuterungsteil veröffentlichte Bewertung der einzelnen Gesellschaften.

76 Vgl. BTDrs. 15/255, S. 21; 15/5550, S. 155.

77 Im Erläuterungsteil des Rechenschaftsberichts für 2003 wird darauf hingewiesen, dass die Überschuldung der Bundespartei und einiger Landesverbände lediglich buchmäßig sei. Aufgrund stiller Reserven im Anlagevermögen sowie bestehender interner Haftungsverhältnisse bestehe bei keinem dieser Verbände eine tatsächliche Überschuldung, BTDrs. 15/ 5550, S. 174.

78 BTDrs. 15/5550, S. 171f.

79 AG Berlin-Charlottenburg, HRB 43013.

80 Dies scheint der Fall zu sein. So werden z.B. die Anteile an der »Universum Trend GmbH«, die als FDP-Unternehmen im Rechenschaftsbericht ausgewiesen ist, von drei Privatpersonen gehalten, AG Wiesbaden, HRB 20831.

81 BTDrs. 15/5550, S. 155, 162. 
Haus- und Grundvermögen weist die FDP 2003 lediglich beim Bundesverband sowie bei den Landesverbänden Bremen, Niedersachsen, Rheinland-Pfalz, SchleswigHolstein und Thüringen aus. Einzelne Immobilien sind mit ihrer Bewertung im Erläuterungsteil aufgeführt. ${ }^{82}$ Darunter sind aber die alte Bonner sowie die neue Berliner Parteizentrale (beide »Thomas-Dehler-Haus « genannt) nicht enthalten. Deren formelle Eigentümerin ist die bereits erwähnte Liberal Vermögensverwaltungs-GmbH \& Co. Vermögensfonds $\mathrm{KG}^{83}$

Bemerkenswert ist die ausdrückliche Erklärung, dass die Jugendorganisation der FDP »Junge Liberale « weder rechtlich noch organisatorisch Teil der Partei sei. Daher seien deren Einnahmen und Ausgaben sowie deren Vermögen nicht im Rechenschaftsbericht enthalten. ${ }^{84}$

\section{e) Bündnis 90/Die Grünen}

Das wirtschaftliche Engagement der Bündnis 90/Die Grünen ist im Vergleich zu den anderen Parteien gering, was auch dem Rechenschaftsbericht 2003 zu entnehmen ist. Die Bundespartei ist erwerbswirtschaftlich nicht tätig, hat keine parteieigenen Unternehmen und ist an anderen Unternehmen nicht beteiligt. Eine Ausnahme ist die Beteiligung an der »taz - Genossenschaft«, die zum 31.12.2004 die Höhe von 10.225,84€ aufwies. ${ }^{85}$ Darüber hinaus sind einige Untergliederungen an Windenergieprojekten beteiligt. ${ }^{86}$ Die ausgewiesenen Gesamteinnahmen der Grünen aus Unternehmenstätigkeit und Beteiligungen beliefen sich 2003 auf die eher bescheidene Summe von 764,29 €.

Der Bundesverband sowie die Landesverbände, die über Immobilienvermögen verfügen, haben zur treuhänderischen Verwaltung von unbeweglichem Vermögen sowie von Forderungen und sonstigen Vermögenswerten Vermögensverwaltungsvereine gegründet. ${ }^{87}$ Mit der fragwürdigen Begründung, dass nach $\S 24$ VII Nr. 3 eine Neubewertung des Haus- und Grundvermögens im Abstand von fünf Jahren vorzunehmen

82 BTDrs. 15/5550, S. 173 f.

83 Das Thomas-Dehler-Haus in Bonn, das dem FDP e.V. gehörte und mit Schulden belastet war, wurde 2003 an die »Liberal Vermögensverwaltungs-GmbH \& Co. Vermögensfonds $\mathrm{KG} \ll$, der auch die Berliner Parteizentrale gehört, veräußert. Für Einzelheiten vgl. Der Spiegel, 27.06.2005; hierzu wurde auf Antrag der Grünen auch eine aktuelle Stunde im Deutschen Bundestag »Steuerrechtliche Positionen der FDP vor dem Hintergrund von Berichten über eigene Finanztransaktionen« durchgeführt, vgl. Deutscher Bundestag, Stenographisches Protokoll, 15. Wahlperiode - 183. Sitzung, 29.06.2005, S. $17283 f$.

84 BTDrs. 15/5550, S. 167.

85 Vgl. D. Strehl, in: Morlok/von Alemann/Streit, Medienbeteiligungen politischer Parteien, Baden-Baden 2004, S. 158. Diese Beteiligung ist aber im Rechenschaftsbericht für das Jahr 2003 nicht aufgeführt (vgl. BTDrs. 15/5550, S. 118), was dadurch erklärlich ist, dass nach $\S 271$ I 5 HGB, auf den § 24 VII Nr. 1 verweist, die Mitgliedschaft in einer eingetragenen Genossenschaft nicht als Beteiligung im Sinne des HGB gilt.

86 Die im Rechenschaftsbericht insoweit genannten Beteiligungen sind ohne Ausnahme geringfügig. Vgl. BTDrs. 15/5550, S. 118.

87 Über Immobilien verfügen die Landesverbände Baden-Württemberg, Brandenburg, Niedersachsen, Nordrhein-Westfalen und Schleswig-Holstein (vgl. BTDrs. 15/2800, S. 95f.). Der Gesamtwert des Haus- und Grundvermögen der Bündnis 90/Die Grünen betrug 2003 11.350.280,93€ (vgl. BTDrs. 15/5550, S. 99). 
sei, wurden im Berichtsjahr 2003, anders als bei den anderen Parteien, keine Neubewertungen vorgenommen. ${ }^{88}$

Auch der Rechenschaftsbericht der Grünen verhält sich zur Rechtslage der Jugendorganisation. Nach der in dieser Form erstmals ausdrücklich so getroffenen begrifflichen Unterscheidung im Rechenschaftsbericht, die an eine verbreitete Terminologie in der parteienrechtlichen Literatur anknüpft, gebe es einerseits Gliederungen der »Grünen Jugend $\ll,{ }^{89}$ die Teilorganisationen der Partei seien, und andererseits solche, die weder rechtlich noch organisatorisch Teil der Partei seien (Nebenorganisationen). Daher seien die Einnahmen und Ausgaben sowie das Vermögen der Letzteren im Parteirechenschaftsbericht nicht enthalten. Wie genau die Abgrenzung zwischen den Teilorganisationen und den Nebenorganisationen verläuft, wird in den Erläuterungen jedoch ebenso wenig geklärt wie die Frage, welche konkret ausgewiesenen Vermögenswerte bzw. Einnahmen nicht der Mutterpartei, sondern den (im Verhältnis zur Partei) »unselbstständigen« Gliederungen der Jugendorganisation zuzuordnen sind.

\section{f) $P D S$}

Der Rechenschaftsbericht 2003 enthält zum ersten Mal konkrete Angaben über das aktuelle Immobilienvermögen sowie über die Unternehmensbeteiligungen der PDS, die vom ehemaligen »Unternehmensimperium « der SED geblieben sind..$^{90}$ Die meisten Unternehmensbeteiligungen der PDS betreffen die Medienbranche und den Immobilienbereich. Direkte Beteiligungen hält die Partei an fünf Parteiunternehmen: »Neues Deutschland Druckerei und Verlag GmbH «, Berlin (50\%), ${ }^{91} »$ Karl Dietz Verlag Berlin GmbH« $(100 \%)$, »Föderative Verlags-, Consulting- und Handelsgesellschaft mbH FEVAC «, Berlin (100\%), »Vulkan Gesellschaft für Grundbesitz $\mathrm{mbH} \ll$, Berlin $(84 \%)^{92}$ und $»$ Hotel am Wald $\mathrm{GmbH} \ll$, Elgersburg $(10,14 \%) .{ }^{93}$ Bemerkenswert ist auch, dass für das Jahr 2003 keine Einnahmen aus Unternehmenstätigkeit und Beteiligungen ausgewiesen wurden. Dafür betragen die Gesamteinnahmen aus sonstigem Vermögen 262.166,32 € und aus Veranstaltungen, Vertrieb von Druckschriften usw. $199.501,71 €^{94}$

Die PDS weist Immobilieneigentum lediglich beim Bundesverband ${ }^{95}$ und beim Landesverband Sachsen-Anhalt aus. Das gesamte Haus- und Grundvermögen der PDS

88 BTDrs. 15/5550, S. 118.

89 BTDrs. 15/5550, S. 116.

90 Die langjährigen gerichtlichen Auseinandersetzungen um das SED-Vermögen endeten mit zwei Vergleichen zwischen der PDS und der Treuhandanstalt, deren Texte in BTDrs. 13/ 10900, Anlage, Dokumente 68 und 69 veröffentlicht sind.

91 AG Berlin-Charlottenburg, HRB 35216, B1. 109.

92 Die übrigen 16\% der Geschäftsanteile werden von der FEVAC gehalten. Die PDS-Anteile werden von Privatpersonen treuhänderisch für die Partei gehalten.

93 Auch hier werden die übrigen Anteile von Parteiunternehmen gehalten. BTDrs. 15/5550, S. 203f.

94 BTDrs. 15/5550, S. 190.

95 Die teuerste Immobilie der PDS ist die Parteizentrale in Berlin (»Karl-Liebknecht-Haus«). Zum Stichtag 21.12.2002 wurde sie mit 4.315.968,78 € bewertet, BTDrs. 15/5550, S. 205. 
wird für 2002 mit 5.374.253,00 € und für 2003 mit 5.164.486,43 € angegeben. ${ }^{96}$ Die Immobilien der PDS sind im Gegensatz zur Praxis anderer Parteien zwar einzeln bewertet und ausgewiesen, jedoch wird auch bei der PDS nicht erläutert, wem konkret (einer Parteigliederung oder einer Vermögensverwaltungsgesellschaft) sie formellrechtlich gehören. Ein Teil der Unternehmensbeteiligungen wird ebenfalls einzeln bewertet und ausgewiesen. ${ }^{97}$

Ebenso wie die FDP postuliert die PDS ausdrücklich, dass ihr Jugendverband rechtlich und organisatorisch nicht Teil der Partei sei und dass daher die Einnahmen und Ausgaben sowie das Vermögen dieser Organisation im Rechenschaftsbericht nicht enthalten seien. ${ }^{98}$

\section{Fazit}

Der Überblick über die ersten Rechenschaftsberichte der Parteien nach der letzten Novelle des Parteiengesetzes zeigt, dass mit den neuen Regelungen wichtige Schritte zur Schaffung größerer Transparenz bezüglich des Parteivermögens, der unternehmerischen Tätigkeit der Parteien und der hieraus resultierenden Einnahmen unternommen worden sind. Die Neuregelungen haben den ersten »Praxistest « jedenfalls im Grundsatz bestanden. Die neueingeführten Bewertungsvorschriften werden künftig die gebotene Vergleichbarkeit der Rechenschaftsberichte der einzelnen Parteien fördern. Ein Einblick in die Immobilienverhältnisse wird jedenfalls ansatzweise gewährleistet. Die vom Gesetzgeber gefundene Lösung bei der Offenlegung der Unternehmensbeteiligungen ist insgesamt ebenfalls positiv zu bewerten. Die Besonderheiten der Medienunternehmen als ein wichtiges Feld parteilicher Wirtschaftstätigkeit werden nunmehr berücksichtigt. Das Parteiengesetz wird diesbezüglich durch die Transparenzvorschriften der Landespressegesetze über das Impressum und die Beteiligungsverhältnisse $^{99}$ ergänzt.

Dennoch bestehen weiterhin Unvollkommenheiten, die die wirksame Offenlegung der Finanzverhältnisse der Parteien behindern.

\section{Problematische Fragen und weiterer gesetzgeberischer Handlungsbedarf}

\section{Die parteinahen Umfeldorganisationen}

Nach wie vor äußerst unbefriedigend ist die Rechtslage bezüglich der Transparenz der wirtschaftlichen Verhältnisse der Hilfsorganisationen der Parteien (etwa der Jugendorganisationen, fachlicher Arbeitsgemeinschaften, aber auch parteinaher Stiftungen) und der finanziellen Beziehungen zwischen diesen Organisationen und der jeweiligen »Mutterpartei«. Alle im Bundestag vertretenen Parteien verfügen über solche Hilfsorganisationen, die für die ideelle und materielle Unterstützung der Parteitätigkeit von

96 Vgl. BTDrs. 15/700, S. 121; 15/2800, S. 143; BTDrs. 15/5550, S. 187.

97 BTDrs. 15/5550, S. 206.

98 BTDrs. 15/5550, S. 199.

99 Vgl. beispielsweise § 7a Berliner Pressegesetz; § 8 Sächsisches Pressegesetz. 
nicht unerheblicher Bedeutung sein dürften. Das Ausmaß der wirtschaftlichen Tätigkeit dieser Organisationen und die Art deren finanzieller Verflechtung mit den »zugehörigen« Parteien kann nicht genau abgeschätzt werden, da den Rechenschaftsberichten diesbezüglich kaum etwas zu entnehmen ist, ${ }^{100}$ was wiederum Folge der äußerst spärlichen gesetzlichen Regelung ${ }^{101}$ dieses Bereiches ist. Möglich ist jedenfalls, dass die Parteien, etwa durch die Auslagerung kostenintensiver Bereiche ihrer Tätigkeit auf bestehende oder eigens zu gründende Organisationen, die dann eigenes Vermögen bilden und alleine oder gemeinsam mit den Mutterparteien oder anderen Hilfsorganisationen eigene Unternehmen gründen können, die gesetzliche Rechenschaftspflicht umgehen.

Es erscheint daher nicht nur sinnvoll, sondern auch geboten, die Rechenschaftspflicht der Parteien in geeigneter Weise auf deren Hilfsorganisationen zu erstrecken. Erstes Desiderat wäre insoweit die Entwicklung einer Legaldefinition der Hilfsorganisation, wobei, wenngleich auch die entgegenstehenden Argumente nicht unbeachtlich sind, einer funktionellen (wirtschaftlichen) Betrachtung ${ }^{102}$ der Vorrang vor einer Definition mit Hilfe formal-organisatorischer Merkmale zu geben sein dürfte. Sodann müssten die Parteien verpflichtet werden, im Rechenschaftsbericht aufzulisten, welche ihrer Umfeldorganisationen sie als Hilfsorganisationen im Sinne des Gesetzes ansehen; deren Vermögenswerte und Wirtschaftstätigkeiten wären in den Rechenschaftsbericht einzubeziehen. ${ }^{103}$ Nimmt eine Partei eine ihrer öffentlich agierenden parteinahen Organisationen nicht in diese Liste auf, so wäre dies im Wege der politischen Debatte und erforderlichenfalls der juristischen Kontrolle korrigierbar. Zudem wäre anders als derzeit - unmissverständlich klar, dass Organisationen, die nicht in der Aufzählung aufgeführt werden, für die jeweilige Partei als »Dritter« zu gelten haben. Damit müssten Geld- oder geldwerte Zuwendungen dieser Organisationen an die Parteien als Spenden in den Rechenschaftsberichten ausgewiesen werden.

100 Zwar machen die Parteien in ihren Rechenschaftsberichten fragmentarische Angaben über ihre Hilfsorganisationen (vgl. BTDrs. 15/5550, S. 32, 70, 116, 167, 199). Sie stellen aber keinesfalls eine ausreichende Grundlage für eine Beurteilung der finanziellen Beziehungen zwischen der Parteiorganisation und den Hilfsorganisationen dar.

101 Das Parteiengesetz enthält eine relevante Regelung nur bezüglich der Jugendorganisationen der Parteien. § 24 XII verlangt von ihnen, die öffentlichen Zuschüsse an ihre Jugendorganisationen im Rechenschaftsbericht nachrichtlich auszuweisen. Bei der Ermittlung der absoluten Obergrenze sind sie ausdrücklich außer Acht gelassen.

102 Rechtsprechung und Lehre haben früh die Gebotenheit einer wirtschaftlichen Betrachtungsweise in Bezug auf die Parteifinanzen erkannt: BGHZ 43, 316 (323); BGHSt 14, 194 (197); OVG Berlin, DVBl. 1992, S. 1301; BVerwGE 92, 196; B. Küstermann (Fußn. 30), S. 235. Der Gesetzgeber ist diesen Erkenntnissen nur teilweise gefolgt, indem § $32 \mathrm{~V}$ PartG für den Fall der Vermögenseinziehung verbotener Parteien auf $\S \S 10-13$ des Vereinsgesetzes verweist, die von einem weiten wirtschaftlichen Vermögensbegriff ausgehen.

103 Vgl. dazu T. Kaufner (Fußn. 20), S. 132; G. Westerwelle, Das Parteienrecht und die politischen Jugendorganisationen, Baden-Baden 1994, S. 145. Zu zurückhaltend sind demgegenüber die Schlussfolgerungen der vom ehemaligen Bundespräsidenten Rau eingesetzten Parteienfinanzierungskommission, vgl. BTDrs. 14/6710, S. $56 f$. 
Die in diesem Zusammenhang geäußerten praktischen und verfassungsrechtlichen Bedenken ${ }^{104}$ überzeugen letztlich nicht. Die Entwicklung von Abgrenzungskriterien mag schwierig sein, ist aber nicht unmöglich, wie etwa ein Blick auf $\S 3$ III VereinsG zeigt. Die Einbeziehung von Hilfsorganisationen in die Rechenschaftspflicht könnte zwar Grundrechte (Art. 14 I, 12 I, 2 I GG) der Parteien bzw. der Hilfsorganisationen berühren. Gerade unter Berücksichtigung des Verfassungsrangs des Transparenzgebots (Art. 21 I 4 GG) wären derartige Einschränkungen jedoch zwanglos zu rechtfertigen. ${ }^{105}$

\section{Ausweis und Bewertung des Parteivermögens}

\section{a) Veröffentlichung einer Liste mit den Immobilien der jeweiligen Partei}

Ohne eigene Immobilien, die Parteieinrichtungen beherbergen, aber auch vermietet werden und als Finanzanlage dienen, kann eine moderne Parteiorganisation schwer funktionieren. Bereits aus den bisher zugänglichen Quellen und nunmehr auch aus den Rechenschaftsberichten ist ersichtlich, dass das Immobilienvermögen der deutschen Parteien beachtlich ist. Qualitativ und quantitativ erschöpfende Angaben hierüber sind aber, wie dargestellt, auch nach der letzten Novelle des Parteiengesetzes ihren Rechenschaftsberichten nicht zu entnehmen. Der Rechnungsposten »Haus- und Grundvermögen « in der Vermögensbilanz wird auch in Zukunft keine ausreichende Auskunft über die Struktur, die Verteilung auf die Parteiuntergliederungen und die Rechtsformen, in denen das Immobilienvermögen der Parteien gehalten wird, geben.

Angesichts der Bedeutung des Immobilieneigentums für die Parteitätigkeit und die Parteifinanzen erscheint eine Präzisierung der gesetzlichen Regelungen sachgerecht. Dabei kommt etwa eine weitere Differenzierung der Vermögensrechnung in Betracht. In Anlehnung an die Vorschläge der Parteienfinanzierungskommission 1983, die gesonderte Posten für Grund und Boden, Gebäude und Außenanlagen sowie Um- und Einbauten in gemieteten Räumen vorgeschlagen hatte, könnte eine weitere Tiefenstaffelung der Rubrik »Haus- und Grundvermögen « vorgesehen werden. ${ }^{106}$ Der Aussagewert dieser bedeutsamen Rubrik könnte dadurch erhöht werden, dass die Parteien zur Veröffentlichung einer Liste aller ihnen wirtschaftlich gehörenden Immobilien im Er-

104 Vgl. H. H. Klein, in: Maunz/Dürig, Grundgesetz, Art. 21, Rn. 237; J. Geerlings, Verfassungs- und verwaltungsrechtliche Probleme bei der staatlichen Finanzierung parteinaher Stiftungen, Berlin 2003, S. 137; B. Küstermann (Fußn. 30), S. 45.

105 Vgl. dazu M. Morlok, in: Dreier, GG, Art. 21, Rn. 42, der zu Recht darauf hinweist, dass das Parteienrecht für diejenigen Organisationen gelten muss, die zur Funktionserfüllung einer Partei unter deren Verantwortung beitragen. Noch herrschend ist eine Ansicht, die, ohne eindeutige Abgrenzungskriterien formaler oder funktionaler Art aufzuzeigen, zwischen Sonder- und Nebenorganisationen unterscheidet. Erstere seien als Teil der jeweiligen Partei, auch in Bezug auf die finanziellen Verhältnisse zu betrachten, letztere dagegen nicht: BVerfGE 2, 1 (78); 5, 85 (392); J. Geerlings (Fußn. 104), S. 136; K.-H. Seifert, Die politischen Parteien im Recht der Bundesrepublik Deutschland, Köln 1975, S. 205.

106 Vom Staat zum Bürger - Bericht zur Neuordnung der Parteienfinanzierung. Vorschläge der vom Bundespräsidenten berufenen Sachverständigenkommission, Köln 1983, S. 184. 
läuterungsteil des Rechenschaftsberichts verpflichtet werden. In dieser »Liegenschaftsliste« wären die einzelnen Immobilien mit Adresse, Baujahr, Nutzungsfläche, Verkehrswert und formellem Eigentümer anzugeben. ${ }^{107}$ Der Gewinn an Transparenz wäre erheblich und der Aufwand für die Parteien nicht unverhältnismäßig groß. ${ }^{108}$

\section{b) Grundbuchfähigkeit politischer Parteien und Typenzwang}

In engem Zusammenhang mit der gerade behandelten Frage steht die im Schrifttum diskutierte Forderung nach einer ausdrücklichen Anerkennung der Grundbuchfähigkeit der Parteien. ${ }^{109}$ Die durch die zivilrechtliche Stellung der Parteien als nichtrechtsfähige Vereine ${ }^{110}$ bedingte Praxis der Eintragung eines Treuhänders bzw. einer Vermögensverwaltungsgesellschaft als Rechtsträger im Grundbuch verschleiert Vermögensverhältnisse der Parteien. ${ }^{111}$ Es wird mit guten Argumenten vertreten, dass der besondere verfassungsrechtliche Status der Parteien die Anerkennung ihrer Grundbuchfähigkeit gebietet, und zwar unabhängig davon, ob sie im Vereinsregister eingetragen worden sind. ${ }^{112}$ Die Rechtsprechung hat erste Schritte in diese Richtung unternommen. ${ }^{113}$ Die ausdrückliche gesetzliche Anerkennung der Grundbuchfähigkeit politischer Parteien als durch die Verfassung hervorgehobene Vereine besonderer Art wäre jedoch vorzugswürdig und läge auf der Linie der Regelung des § 24 VI Nr. 1 A I 1, der die Parteien verpflichtet, in ihren Rechenschaftsberichten ihr »Haus- und Grundvermögen« anzugeben, was trotz der Verwendung des Begriffs »Vermögen« statt »Eigentum « letztlich nur dann sinnvoll erscheint, wenn die Parteien selbst im Grundbuch eingetragen werden können.

Ernsthaft erwogen werden sollte in diesem Zusammenhang die Einführung einer gesetzlichen Regelung der Art, dass politische Parteien generell als eingetragene Vereine zu organisieren und nicht nur für die Zwecke des Grundbuchrechts als solche zu behandeln sind. Die historischen Gründe, die die Parteien gezwungen hatten, sich als nichtrechtsfähige Vereine zu konstituieren, ${ }^{114}$ sind längst entfallen, das gewisse »Chaos « bei der Rechtsform deutscher politischer Parteien stellt somit einen Anachronismus dar. Zwar wird im Schrifttum nahezu einmütig an der Auffassung festgehalten, gegen eine gesetzliche Pflicht für die Parteien, sich als rechtsfähige Vereine zu organisieren oder sich in einem Parteienregister mit deklaratorischer Wirkung registrieren zu

107 Ähnlich J. Rübenkönig, Die Rechenschaftspflicht der politischen Parteien nach Art. 21 Absatz 4 Grundgesetz, Frankfurt am Main 2003, S. 209.

108 Vgl. I. Wettig-Danielmeier, ZParl 2001, S. 528 (535).

109 Vgl. M. Morlok/ A. Schulze-Trux, NJW 1992, S. 2058 (2060); Konzen, JuS 1989, S. 20 (24); Schmidt, NJW 1984, S. 2249 (2251).

110 Die meisten Parteien sind als nichtrechtsfähige Vereine konstituiert und sind als solche nach herrschender Auffassung nicht grundbuchsfähig. Für eine Gegenansicht vgl. K. F. Kempfler, NJW 2000, S. 3763; Reuter, in: MünchKomm, § 54, Rn. 29.

111 Die rechtliche Bindung des jeweiligen Treuhänders bzw. der Handelsgesellschaft an eine Partei ist aus dem Grundbuch nicht ersichtlich, weil es Hinweise lediglich auf dinglich wirkende Rechtsgeschäfte enthält.

112 Vgl. M. Morlok/A. Schulze-Trux (Fußn. 109), S. 2061; H. Konzen, JuS 1989, S. 20 (24).

113 Vgl. OLG Zweibrücken, NJW-RR 2000, S. 749; OLG Celle, NJW 2004, S. 1743.

114 Vgl. K. Endlich (Fußn. 28), S. 7f. 
lassen, bestünden durchgreifende verfassungsrechtliche Bedenken. ${ }^{115}$ Hierbei handelt es sich jedoch lediglich um historisch bedingte Reflexe, die keine Stütze im Verfassungstext und der Verfassungswirklichkeit finden. Die Rechtsform des rechtsfähigen Vereins wäre der ungehinderten Teilnahme der Parteien am Geschäftsverkehr dienlich. Die Herstellung klarer Rechtsverhältnisse würde der Argumentation, aus »Rechtsgründen« seien wirtschaftlich undurchschaubare Vermögensschachtelungen erforderlich, den Boden entziehen. ${ }^{116}$

\section{c) Offenlegung von Treuhandverhältnissen}

Angesichts dessen, dass auch Parteien, die bereits jetzt als rechtsfähige Vereine konstituiert sind, Treuhandkonstruktionen benutzen, ist jedoch fraglich, ob die Parteien nach einer gesetzlichen Anerkennung ihrer Grundbuchfähigkeit darauf verzichten würden. Daher sollte eine solche Regelung durch die gesetzliche Beschränkung der Treuhandverhältnisse der Parteien ergänzt werden. Wie bereits ausgeführt hat die Praxis der Einsetzung von Treuhändern nämlich eine erhebliche Minderung der Transparenz des Immobilienvermögens und der unternehmerischen Tätigkeit der Parteien zur Folge. Ein Verbot der Einsetzung von Treuhändern wäre zum gegenwärtigen Zeitpunkt unzulässig, da die als nichtrechtsfähige Vereine verfassten Parteien auf Treuhänder angewiesen sind. Nach einer Anerkennung der Grundbuchfähigkeit von Parteien könnte diese Frage anders $\mathrm{zu}$ beurteilen sein. Weniger weitgehend und rechtspolitisch wohl auch ausreichend, aber auch notwendig, erscheint demgegenüber ein Gebot der Offenlegung aller Treuhandverhältnisse im Rechenschaftsbericht.

\section{d) Effektivere Offenlegung der mittelbaren Unternehmensbeteiligungen}

Angesichts der aufgezeigten Unvollkommenheiten bei der Publizität der mittelbaren Unternehmensbeteiligungen der Parteien ist die effektivere Offenlegung von Beteiligungsketten erforderlich. Da die derzeitige Rechenschaftspraxis die Zuordnung der mittelbaren Beteiligungen zum jeweiligen Parteiunternehmen nicht erlaubt, sollten die Parteien zu entsprechenden Angaben verpflichtet werden. Andernfalls bleiben die Rechenschaftsberichte in diesem Punkt unübersichtlich und nicht ausreichend informativ. Denn Angaben über die konkrete Struktur der Parteibeteiligungen können für das Erkennen wirtschaftlicher Interessen und Abhängigkeiten einer Partei durchaus bedeutsam sein.

115 Beispiele für die argumentationslose Tabuisierung einer Regelung der Rechtsform der Parteien und einer Parteienregistrierung R. Streinz, in: Mangoldt/Klein/Starck, Art. 21, Rn. 51; M. Morlok, in: Dreier, GG, Art. 21 Rn. 32 und 57; J. Ipsen, in: Sachs, GGK, Art. 21, Rn. 27. Für ein Registrierungsverfahren hingegen W. Wietschel, Der Parteibegriff, BadenBaden 1996, S. $195 f$.

116 Die Einführung eines Parteienregisters hätte weitere Vorteile. So würde die Notwendigkeit entfallen, dass wie derzeit verschiedene Stellen, die parteirelevante Entscheidungen zu treffen haben, unabhängig voneinander die Erfüllung der Merkmale des Parteibegriffs prüfen, was die Rechtssicherheit erhöhen und bürokratische Arbeit vermindern würde. $\mathrm{Zu}$ bemerken ist, dass eine Art Parteienregistrierung im Grunde bereits besteht, und zwar beim Bundeswahlleiter (§ 6 III PartG). 


\section{Ausweis und Bewertung der Einnahmen aus Parteivermögen und unternehmeri- scher Tätigkeit}

\section{a) Gesonderter Ausweis der Mieteinnahmen}

Im Parteiengesetz ist eine gesonderte Ausweisung der Einnahmen aus der Nutzung von Immobilien nicht vorgesehen. Die Mieteinnahmen werden von den einzelnen Parteien uneinheitlich als Einnahmen aus sonstigem Vermögen (§ 24 IV Nr. 6) oder als sonstige Einnahmen (Nr. 9) ausgewiesen. ${ }^{117}$ Die Parteien geben ungern konkrete Angaben über die Höhe ihrer Mieteinnahmen. Lediglich Bündnis 90/Die Grünen weisen regelmäßig in ihren Rechenschaftsberichten ihre Mieteinnahmen aus. ${ }^{118}$

Die Einführung einer gesonderten Position »Mieteinnahmen« im Rechenschaftsbericht erscheint nicht nur deswegen geboten, weil das Haus- und Grundvermögen regelmäßig einen großen Anteil am Gesamtparteivermögen hat. Die Information darüber, wie eine Partei ihre Immobilien nutzt, wem sie ihre Gebäude vermietet und in welcher Höhe Mieteinnahmen daraus erzielt werden, könnte für das Urteil des Wählers ebenso wichtig sein wie die Kenntnis über die Herkunft und Höhe von Parteispenden. Darüber hinaus begünstigt das »Zwielicht «, in dem die Anmietung und Vermietung von Immobilien derzeit stattfindet, »Quersubventionierungen« aller Art über den Weg marktunüblich hoher oder niedriger Mietzinsen. Eine gesonderte Position für die Mieteinnahmen könnte in einem Zuge mit der unter V.2.a. bezeichneten »Immobilienliste« eingeführt werden.

\section{b) Ausweis der Erträge aus Unternehmensbeteiligungen}

Die globale Ausweisung der Einnahmen aus unternehmerischer Tätigkeit unter der Position »Einnahmen aus Unternehmenstätigkeit und Beteiligungen« erscheint hinsichtlich der Bedeutung der Parteiunternehmen für die Finanzierung und die allgemeine politische Tätigkeit der Parteien und des notwendigen Einblicks in ihre Finanzstruktur unbefriedigend. Für eine wirkliche Transparenz der Einnahmen aus Unternehmensbeteiligungen wären Angaben über die Dividendenausschüttung an die Partei je Einzelunternehmen notwendig. Dies könnte auch für die Bewertung des aktuellen Wertes einzelner Unternehmen hilfreich sein. Eine gesonderte Offenlegung der Gewinnabführungen einzelner Unternehmen würde ebenfalls den Zusammenhang mit der Auflistung der einzelnen Beteiligungen im Erläuterungsteil der Vermögensbilanz erleichtern. Eine Pflicht zur Ausweisung jeder einzelnen Gewinnausschüttung unabhängig von der Höhe erschiene jedoch überzogen, da dies die Übersichtlichkeit der Rechenschaftsberichte beeinträchtigen und zu unvertretbarem Aufwand für die Parteien führen würde. Die Lösung könnte hier in der Festsetzung eines Schwellenwertes liegen. Bezüglich der Festlegung des Betrags und der konkreten Formulierung der einzuführenden Vorschrift bietet sich eine Parallelität zu den spendenbezogenen Regelungen des $\S 25$ III 2 und 3 an.

117 Vgl. BTDrs. 14/5050, S. 82; 14/2508, S. 108; 14/5050, S. 109; 15/2800, S. 96.

118 Vgl. BTDrs. 14/8022, S. 103f.; 15/2800, S. 96f.; 15/700, S. 79f. 


\section{c) Der Rechnungsposten »sonstige Einnahmen«}

Der Rechnungsposten »sonstige Einnahmen« (§ 24 IV Nr. 9) erwies sich früher oft als »Einfallstor « für die Einschleusung illegaler Einnahmen ins Parteivermögen. ${ }^{119}$ Unter diesem Punkt sind Einnahmen auszuweisen, die keinen Bezug zu den anderen Einnahmenarten aufweisen und sich nicht den anderen Einnahmeposten zuordnen lassen. ${ }^{120}$ Die Handhabung dieser Position ist von Partei zu Partei sehr unterschiedlich, was der Transparenz abträglich ist. ${ }^{121}$ Dies gilt insbesondere für die Offenlegung der Mieteinnahmen, die de lege lata wegen ihres direkten Bezugs zum Haus- und Grundvermögen richtigerweise als Einnahmen aus Vermögen und nicht als sonstige Einnahmen auszuweisen sind. ${ }^{122}$ Gemäß $§ 27$ II 2 sind Einnahmen, die im Einzelfall die Summe von $10.000,-€$ übersteigen, offen zu legen. Diese Vorschrift bezieht sich nach richtiger Auffassung nur auf die »sonstigen Einnahmen«. Die Gegenansicht ${ }^{123}$ lässt die Gesetzessystematik und den Sinn und Zweck der Norm außer Acht.

$\mathrm{Ob}$ auch hinsichtlich der gesetzlichen Regelung dieses Rechnungspostens weiterer selbstständiger Reformbedarf besteht, bleibt abzuwarten. Von Interesse wird insbesondere sein, ob künftige Rechenschaftsberichte unter diesem Punkt auffallend hohe und nicht plausibel aufgeschlüsselte Beträge verzeichnen. Als Auffangkategorie wird der Posten in jedem Falle erforderlich bleiben.

119 Es sei an die Praxis der hessischen CDU erinnert. Vgl. dazu BTDrs. 14/9300, S. 220f.; BVerfG, DVB1. 2004, S. 1357f.

120 Vgl. BTDrs. 14/4747, S. 24. Unter diesem Posten werden üblicherweise Schadenersatzleistungen, Kostenerstattungen von Dritten, Mieteinnahmen, Erbschaften und Vermächtnisse, staatliche Zuschüsse an Parteijugendorganisationen sowie Lohnkostenzuschüsse nach dem SGB III usw. aufgeführt. Die Existenz dieser Auffangkategorie ist finanztechnisch notwendig, vgl. D. Strehl, in: Wettig-Danielmeier/Stiegler, Reform der Parteienfinanzierung, Marburg 2001, S. 56 sowie J. Rübenkönig (Fußn. 107), S. 176.

121 Vgl. zu den sonstigen Einnahmen der FDP (BTDrs. 11/3315, S. 70), der CDU (13/8888, S. 23; 15/2800, S. 58), der PDS (14/2508, S. 129; 15/7700, S. 156). Bemerkenswert ist, dass manche Parteien unter dieser Position oft erhebliche Beiträge ausweisen (vgl. z.B. BTDrs. 14/5050, S. 4 - SPD -, S. 46 - CDU -, S. 84 - Bündnis 90/Die Grünen - und BTDrs. 14/8022, S. 2 - SPD -, S. 42 - CDU -, S. 130 - FDP -). Vgl. auch die Angaben bei T. Kaufner (Fußn. 20), S. 114.

122 De lege ferenda vgl. oben 3.1.

123 C. Lenz (Fußn. 36), S. 772. 